\title{
Mechanisms and convergence of compensatory evolution in mammalian mitochondrial tRNAs
}

\author{
Andrew D Kern \& Fyodor A Kondrashov
}

The function of protein and RNA molecules depends on complex epistatic interactions between sites. Therefore, the deleterious effect of a mutation can be suppressed by a compensatory second-site substitution ${ }^{1,2}$. In relating a list of 86 pathogenic mutations in human tRNAs encoded by mitochondrial genes to the sequences of their mammalian

orthologs, we noted that 52 pathogenic mutations were present in normal tRNAs of one or several nonhuman mammals. We found at least five mechanisms of compensation for 32 pathogenic mutations that destroyed a Watson-Crick pair in one of the four tRNA stems: restoration of the affected WatsonCrick interaction (25 cases), strengthening of another pair (4 cases), creation of a new pair (8 cases), changes of multiple interactions in the affected stem (11 cases) and changes involving the interaction between the loop and stem structures (3 cases). A pathogenic mutation and its compensating substitution are fixed in a lineage in rapid succession, and often a compensatory interaction evolves convergently in different clades. At least $10 \%$, and perhaps as many as $50 \%$, of all nucleotide substitutions in evolving mammalian tRNAs participate in such interactions, indicating that the evolution of tRNAs proceeds along highly epistatic fitness ridges.

We mapped 86 human pathogenic mutations in the 22 tRNAs encoded by mitochondrial genes, many of which are known to cause serious disease early in life ${ }^{3,4}$, on the corresponding alignments of orthologous tRNAs from 106 mammalian species. Often a site in the nonhuman tRNA was occupied by a nucleotide that represents a pathogenic mutation at the homologous human site, a pattern called a compensated pathogenic deviation ${ }^{2}$ (CPD; Fig. 1). An organism carrying a CPD must also have some other, compensatory deviation from humans that suppresses the deleterious effect of the $\mathrm{CPD}^{2,5}$. CPDs are common in proteins encoded by nuclear genes, but the molecular mechanism of compensation has been determined for only a small fraction of them ${ }^{2,5}$. Here, we systematically analyzed the mechanisms of intramolecular compensation in the evolution of mammalian mitochondrial tRNAs.

We identified 52 CPDs that occurred 1,207 times in 105 mammalian species, including 90 occurrences of 27 CPDs in 12 primate species (Table 1; alignments in Supplementary Sequence Archive online). These CPDs probably do not represent sequencing errors, because different species often carry the same CPD (Fig. 1 and Supplementary Sequence Archive online) and no CPDs were found at polymorphic sites in species with multiple completely sequenced mitochondria (data not shown). The CPDs are also probably not a result of errors in the Mitomap data set. First, pathogenic mutations are found in tRNA sites that are much more conservative than sites that are known to be polymorphic in humans ${ }^{6}$ (Supplementary Table 1 online). Second, many pathogenic mutations are well characterized, so that errors in Mitomap must be rare $^{3,4}$, whereas $60 \%$ of the mutations in the database were found as a CPD. Last, a subset of the best characterized pathogenic mutations, which are labeled as being confirmed in Mitomap, showed a similar proportion of CPDs (data not shown).

The secondary structure of most mitochondrial tRNAs is that of a cloverleaf with four stems and three loops ${ }^{7}$. Most CPDs (34) were found in stem structures, and 32 of them disrupted a Watson-Crick pair. Therefore, we assumed that substitutions compensating for the deleterious effect of pathogenic mutations usually reverse the loss of Gibbs free energy in stem structures ${ }^{8}$. Studies of compensation of substitutions that disrupt Watson-Crick pairs emphasized second-site substitutions that restore the disrupted Watson-Crick interaction ${ }^{9-14}$. This mechanism of compensation is common (Table 2). For example, in the anticodon stem of human tRNA ${ }^{\text {Asn }}$, the mutation C27U (G5703A in mitochondrial genome notation) destroys a GC pair. In chimpanzees (Pan troglodytes), this Watson-Crick interaction is restored by a G43A substitution that creates an AU pair (Fig. 2a). Species that are more distant from humans may have multiple CPDs in the same tRNA (Fig. 1); for example, the Malayan flying lemur (Cynocephalus variegatus) has evolved compensations of three pathogenic mutations in $\mathrm{tRNA}^{\text {Lys }}$, each through the direct restoration of the disrupted Watson-Crick interaction (Fig. 2b).

We also noted three indirect mechanisms of compensation of CPDs that disrupt Watson-Crick pairs, which involve substitutions at sites that do not pair directly with that of the CPD. In the white rhinoceros (Ceratotherium simum), the pathogenic human mutation G30A (G5540A) that disrupts a GC pair in the anticodon stem of tRNA ${ }^{\operatorname{Trp}}$ is compensated by a substitution that creates an extra Watson-Crick 
a Homo sapiens (human)

Pan troglodytes (chimpanzee)

Pan paniscus (pygmy chimpanzee)

Gorilla gorilla (gorilla)

Pongo pygmaeus (orangutan)

Pongo pygmaeus abelii (Sumatran orangutan)

Papio hamadryas (hamadryas baboon)

Macaca sylvanus (Barbary ape)

Hylobates lar (common gibbon)

Cebus albifrons (white-fronted capuchin)

Lemur catta (ring-tailed lemur)

Nycticebus coucang (slow loris)

Tarsius bancanus (western tarsier)

b

Homo sapiens (human)

Tarsius bancanus (western tarsier)

Tupaia belangeri (northern tree shrew)

Lepus europaeus (European hare)

Jaculus jaculus (lesser Egyptian jerboa)

Sciurus vulgaris (Eurasian red squirrel)

Echinops telfairi (small Madagascar hedgehog)

Pteropus scapulatus (little red flying fox)

Pipistrellus abramus (Japanese house bat)

Ursus maritimus (polar bear)

Odobenus rosmarus rosmarus (Atlantic walrus)

Rhinoceros unicornis (greater Indian rhinoceros)

Monodon monoceros (narwhal)

Platanista minor (Indus River dolphin)

Sus scrofa (pig)

Dasypus novemcinctus (nine-banded armadillo)

Orycteropus afer (aardvark)

Elephas maximus (Asiatic elephant)

Macropus robustus (wallaroo)

Vombatus ursinus (common wombat)

Ornithorhynchus anatinus (platypus)

Tachyglossus aculeatus (Australian echidna)
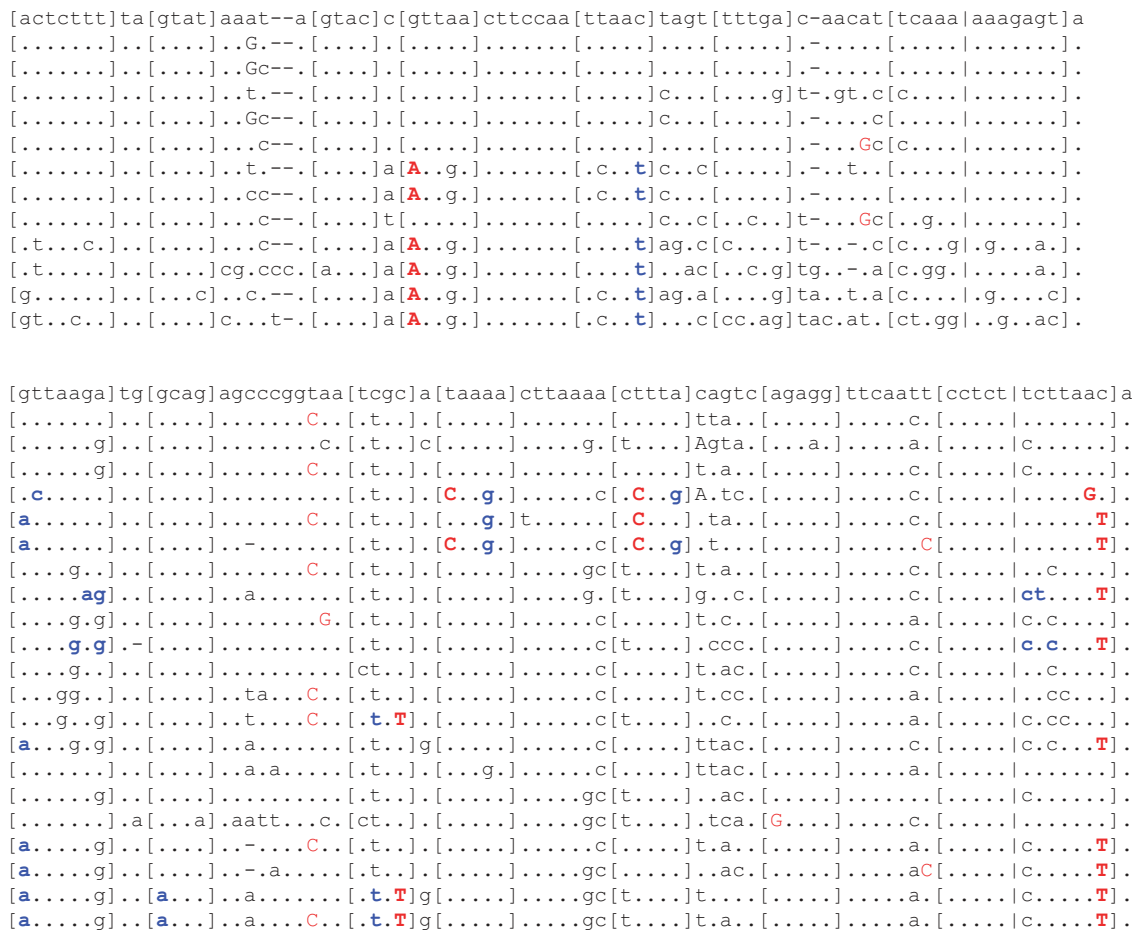

Figure 1 Multiple alignments of tRNAs with secondary structure annotation. (a) Genes encoding primate tRNAGly (b) Selected nonprimate mammalian gene

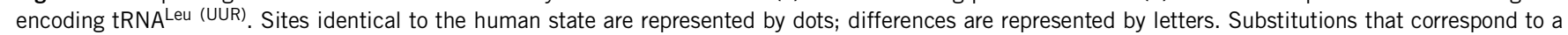
human pathogenic mutation (CPD) are capitalized and shown in red; the compensatory substitutions are shown in blue. CPDs with identified compensatory substitutions are in bold. Sequence corresponding to stem structures is in brackets.

pair in the same stem (Fig. 2c), thus reversing the loss of free energy ${ }^{8}$. Compensation can also occur through strengthening of an existing Watson-Crick pair, which requires two substitutions. In the polar bear (2) (Ursus maritimus), replacement of an AU pair with a stronger GC pair abolishes the deleterious effect of the mutation A5G that converts a Watson-Crick pair into a weak GU pair in the acceptor stem of tRNA $^{\text {Ser }}$ (Fig. 2d). The same GC pair is also involved in compensation of the $\mathrm{A} 3 \mathrm{G}$ pathogenic mutation, indicating that compensatory evolution may involve complex, epistatic interactions between multiple sites. Finally, multiple changes can compensate for one pathogenic mutation. The loss of a GC pair in the D stem of tRNA ${ }^{\text {Leu (UUR) }}$ is compensated by the creation of two new, weaker pairs on the other end of the stem in the northern bottlenose whale (Hyperoodon ampullatus; Fig. 2e). The Australian echidna (Tachyglossus aculeatus) compensates for the same mutation directly, by replacing the disrupted GC pair with a weaker $\mathrm{AU}$ pair, and the evolution of another $\mathrm{AU}$ pair in a different part of the D stem may also aid the compensation (Fig. 2f). Different species may compensate for the same pathogenic mutation by different mechanisms. For example, Ehrenberg's mole rat (Spalax ehrenbergi) has evolved a new GC pair in the D stem of tRNA ${ }^{\text {Ile }}$ that compensates for the loss of a neighboring AU pair (Fig. 2g). The southern tamandua (Tamandua tetradactyla), in contrast, compensates for the same CPD by direct restoration of Watson-Crick interaction (Fig. $\mathbf{2 h}$ ).

Another mechanism of compensation involves the interaction of stem and loop structures. For example, the loss of an AU pair in the D stem of rabbit (Oryctolagus cuniculus) tRNA ${ }^{\text {Cys }}$ is compensated for by elongation of the D loop and elimination of the hairpin loop found in human tRNA ${ }^{\text {Cys }}$ (Fig. 3a). Similarly, compensation for a CPD in a loop may involve compensations in the corresponding stem structure.
We observed 18 CPDs in loops (Fig. 2e-h), but a comprehensive analysis of the tertiary interactions involved must be deferred until crystal structures of the tRNAs become available ${ }^{15}$. In one instance we identified the substitutions involved in compensating for the pathogenic mutation A3243G in the D loop of tRNA ${ }^{\text {Leu (UUR) }}$. tRNAs with an A3243G mutation are prone to forming a six-nucleotide dimer between two tRNAs, which requires a partial denaturation of the $D$ stem ${ }^{15,16}$, suggesting that strengthening the $\mathrm{D}$ stem may prevent the pathogenic effect of the $\mathrm{A} 3243 \mathrm{G}$ mutation ${ }^{15}$. The $\mathrm{A} 3243 \mathrm{G}$ mutation is fixed in dogs (Canis familiaris), and they probably retain a normal phenotype because of strengthening of the D stem through the creation of two new Watson-Crick bonds (Fig. 3b).

For the 32 CPDs that destroy a Watson-Crick pair, we noted 25 instances of direct restoration of the Watson-Crick pairing of the affected site (Table 2), indicating that different Watson-Crick pairs are often functionally interchangeable. We also found 26 cases of indirect compensation of these CPDs by one of the three mechanisms described above (Table 2), which indicates that different organisms often compensate for the same CPD in different ways. In the remaining seven cases, we could not determine what compensates for the CPD (this was also the case for two within-stem CPDs that do not destroy a Watson-Crick pair). Thus, for each pathogenic mutation in a stem, there seem to be more than two potential methods of compensation, one involving restoration of the Watson-Crick interaction (available for almost all mutations) and, on average, more than one indirect change. Thus, most compensatory evolution in tRNAs occurs through complex, indirect mechanisms, indicating that models that consider only compensation that restores Watson-Crick pairs are oversimplified ${ }^{9-14,17}$. 
Table 1 Occurrences of CPDs across different mammalian species for different mitochondrial tRNAs

\begin{tabular}{|c|c|c|c|c|c|c|}
\hline tRNA & $\begin{array}{l}\text { Number } \\
\text { of CPDs } \\
\text { in mammals } \\
\text { (primates) }\end{array}$ & $\begin{array}{l}\text { Number of } \\
\text { different CPDs } \\
\text { across } \\
\text { mammalian } \\
\text { (primate) } \\
\text { species }\end{array}$ & $\begin{array}{c}\text { Number of } \\
\text { pathogenic } \\
\text { mutations } \\
\text { known }\end{array}$ & $\begin{array}{l}\text { Number of } \\
\text { CPD } \\
\text { sites with } \\
\text { known } \\
\text { (unknown) } \\
\text { compensations* }\end{array}$ & $\begin{array}{l}\text { Number of } \\
\text { evolutionarily } \\
\text { independent } \\
\text { CPDs with } \\
\text { known } \\
\text { (unknown) } \\
\text { compensations* }\end{array}$ & $\begin{array}{c}\text { Number of } \\
\text { evolutionary } \\
\text { independent } \\
\text { compensatory } \\
\text { substitutions* }\end{array}$ \\
\hline Ala & $9(1)$ & $1(1)$ & 1 & 1 & 5 & 5 \\
\hline Cys & $4(2)$ & $1(1)$ & 1 & 1 & 4 & 4 \\
\hline Asp & $7(2)$ & $1(1)$ & 1 & 1 & 4 & 4 \\
\hline Glu & $6(2)$ & $1(1)$ & 1 & $0(1)$ & $0(5)$ & 0 \\
\hline Phe & $166(11)$ & $3(2)$ & 4 & 3 & 5 & 5 \\
\hline Gly & $89(12)$ & $4(3)$ & 5 & $2(2)$ & $8(4)$ & 7 \\
\hline His & $81(9)$ & $2(1)$ & 2 & $1(1)$ & $5(1)$ & 5 \\
\hline Ile & $68(7)$ & $5(2)$ & 10 & $1(4)$ & $3(16)$ & 2 \\
\hline Lys & $201(9)$ & $5(3)$ & 12 & $4(1)$ & $16(1)$ & 21 \\
\hline Leu (UUR) & $77(8)$ & $11(3)$ & 18 & $6(5)$ & $11(18)$ & 13 \\
\hline Leu (CUN) & $15(1)$ & $3(1)$ & 5 & $1(1)$ & $1(2)$ & 1 \\
\hline Met & $0(0)$ & $0(0)$ & 2 & 0 & 0 & 0 \\
\hline Asn & $95(1)$ & $2(1)$ & 3 & $1(1)$ & $1(1)$ & 1 \\
\hline Pro & $0(0)$ & $0(0)$ & 1 & 0 & 0 & 0 \\
\hline Gln & $13(3)$ & $1(1)$ & 2 & $0(1)$ & $0(7)$ & 7 \\
\hline Arg & $0(0)$ & $0(0)$ & 0 & 0 & 0 & 0 \\
\hline Ser (UCN) & $214(13)$ & $5(3)$ & 5 & $4(1)$ & $11(1)$ & 7 \\
\hline Ser (AGY) & $2(1)$ & $1(1)$ & 2 & 0 & 0 & 0 \\
\hline Thr & $25(1)$ & $2(1)$ & 3 & 1 & 8 & 2 \\
\hline Val & $132(7)$ & $3(1)$ & 4 & 3 & 8 & 8 \\
\hline Trp & $3(0)$ & $1(0)$ & 3 & 1 & 2 & 2 \\
\hline Tyr & $0(0)$ & $0(0)$ & 1 & 0 & 0 & 0 \\
\hline Total & $1,207(90)$ & $52(27)$ & 86 & $31(18)$ & 99 (49) & 94 \\
\hline
\end{tabular}

*Only in species that were included in the mammalian phylogeny ${ }^{18}$.

We obtained predictions for the compensatory substitutions by visually selecting a candidate substitution and then predicting its effect on the free energy of the tRNA molecule using mfold ${ }^{8}$ (Supplementary Methods online). In a few instances the CPD-containing stem structures had both a substitution that restored the disrupted WatsonCrick pair and one or more other substitution(s) that restored the free energy of the stem. In such cases we classified the compensation only as a Watson-Crick restoration event. Thus, our estimate of the involvement of indirect compensation is conservative because these other substitutions may also contribute to compensation, especially when direct compensation involves replacement of an $\mathrm{AU}$ pair with a GC pair (that is, a weaker with a stronger interaction).

We observed high rates of convergent evolution in substitution pairs the compensate for CPDs among the mammalian taxa examined (Fig. 4 and Table 1). For the 69 species in a recent mammalian phylogeny ${ }^{18}$, we found that on average, a CPD evolved independently three times (Table 1). This high incidence is probably not a consequence of fast evolution of mitochondrial DNA, because mammalian tRNAs are generally conservative and even monotreme tRNAs are very similar to their placental orthologs (Fig. $\mathbf{1 b}$ and Supplementary Sequence Archive online). CPDs and compensatory substitutions evolve in quick succession in an apparently dependent way such that a CPD is rarely found without the compensatory substitution (Fig. 4). Almost every independent evolutionary event leading to the fixation of a $\mathrm{CPD}$ is directly associated with a unique origin of a compensatory change (Table 1).

A pathogenic mutation in a mitochondrial tRNA is unlikely to be fixed without a compensatory change, because the fitness of an organism with such a mutation is nearly zero $^{2}$. Thus, there are only two scenarios for the evolution of a CPD and the corresponding compensatory substitution. In the simpler scenario, the compensatory substitution is the first to achieve fixation, with the CPD becoming fixed afterwards ${ }^{2}$. Alternatively (and perhaps less probably), the CPD and the compensatory substitution achieve fixation simultaneously. This may be possible if the pathogenic mutation is maintained in the population at a very low frequency and the compensatory substitution occurs in an individual with the

Table 2 The molecular basis of compensatory evolution in mitochondrial tRNAs

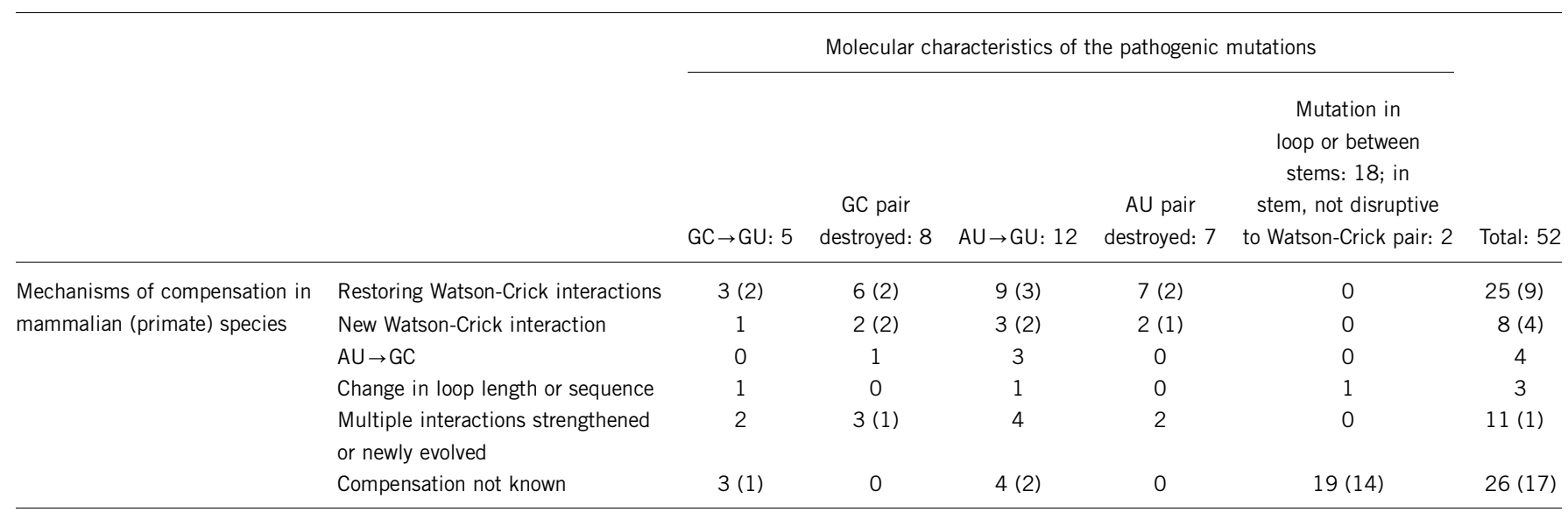

Columns list the characteristics of the pathogenic mutations (CPDs); rows list the characteristics of the compensatory substitutions. 
a

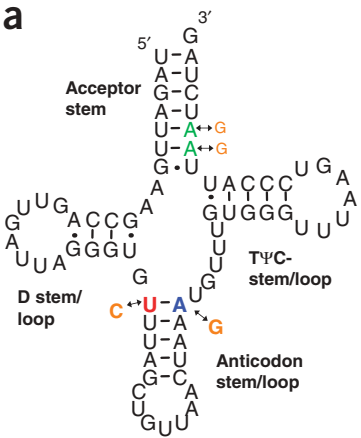

e

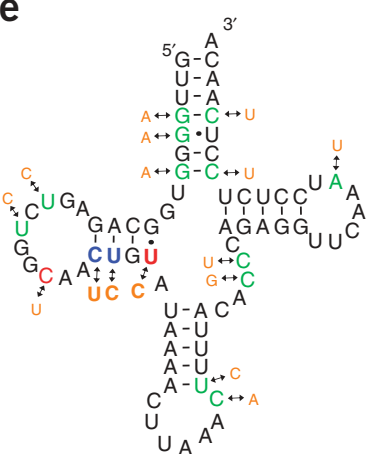

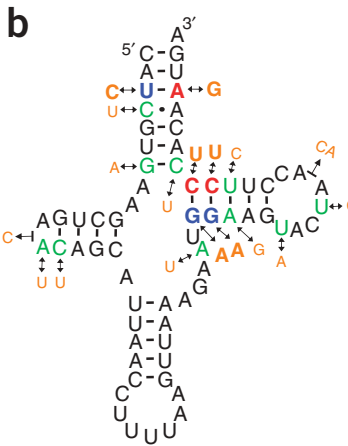

f

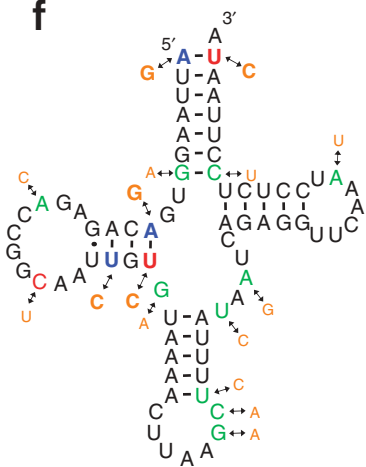

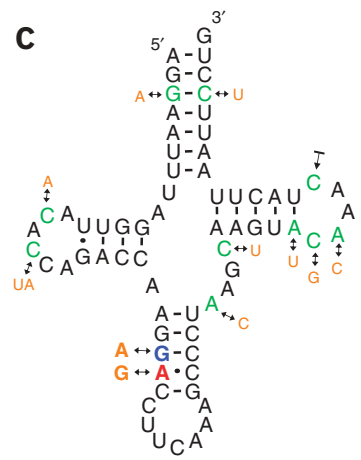
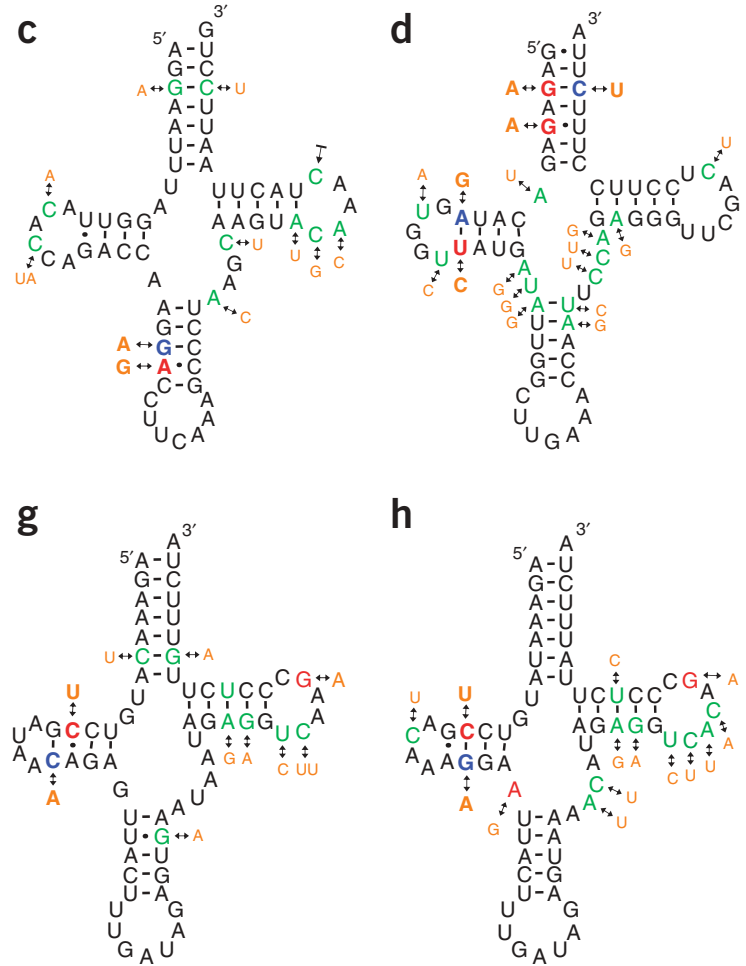

h

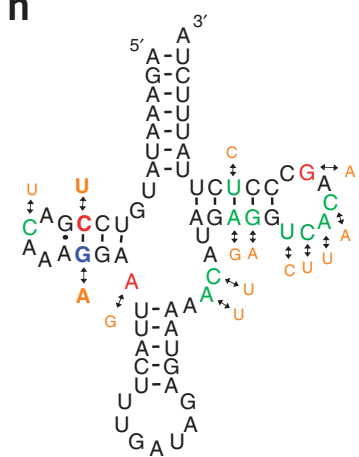

Figure 2 Secondary structures of selected nonhuman tRNAs. (a) P. troglodytes (chimpanzee) tRNA Asn. (b) C. variegatus (Malayan flying lemur) tRNALys. (c) C. simu (white rhinocerus) tRNATrp. (d) U. maritimus (polar bear) tRNA Ser (UCN). (e) H. ampullatus (northern bottlenose whale) tRNA ${ }^{\text {Leu (UUR) }}$. (f) T. aculeatus (Australian echidna) tRNA ${ }^{\text {Leu (UUR) }}$. (g) S. ehrenbergi (Ehrenberg's mole rat) tRNA ${ }^{\text {Ile }}$. (h) T. tetradactyla (southern tamandua) tRNA ${ }^{\text {lle }}$. CPDs (nucleotides corresponding to human pathogenic mutations) are shown in red; predicted compensatory substitutions are shown in blue; and other deviations from the human ortholog, those unrelated to the pathogenic mutations or their compensations, are shown in green. Nucleotides found in healthy humans are shown in orange alongside the nonhuman sequence.

CPD with subsequent fixation of the resulting haplotype ${ }^{19}$. The latter scenario is perhaps more likely to occur in mitochondrial than in nuclear genes, because mitochondrial pathogenic mutations may persist in a heteroplasmic state.

The high rate of compensatory evolution and the coupling of the evolution of CPDs and their compensations may indicate that positive selection is involved in the evolution of compensatory interactions ${ }^{2,20}$. Positive selection would reduce the time between the fixations of a compensatory change and the corresponding CPD, explaining the simultaneous convergent evolution of CPDs and the compensatory substitutions. In the absence of doublet mutations, the fraction of CPDs in diverging species is expected to increase if the accumulating differences evolve neutrally by genetic drift ${ }^{21,22}$. In contrast, positive selection should lead to a more constant proportion of compensatory substitutions on molecular distance ${ }^{2}$. This prediction holds true for primate species, such that the proportion of CPDs among all deviations is constant across molecular and phylogenetic distances (Supplementary Fig. 1 online). Thus, the fixation of the compensatory substitutions or of the CPD-compensatory haplotype in mitochondrial tRNAs occurs as a result of positive selection.

The list of human mitochondrial pathogenic mutations is far from complete ${ }^{3}$. Therefore, our observation that $5 \%$ of all substitutions are CPDs or, assuming that each CPD interacts with one compensatory substation, $10 \%$ of all substitutions participate in a compensatory interaction (Supplementary Fig. 1 online) is a minimum estimate. Assuming that tRNA ${ }^{\text {Leu (UUR) }}$ is representative of an average tRNA, we estimate that $25 \%$ of all substitutions are CPDs, with another $25 \%$ corresponding to compensatory substitutions (Supplementary
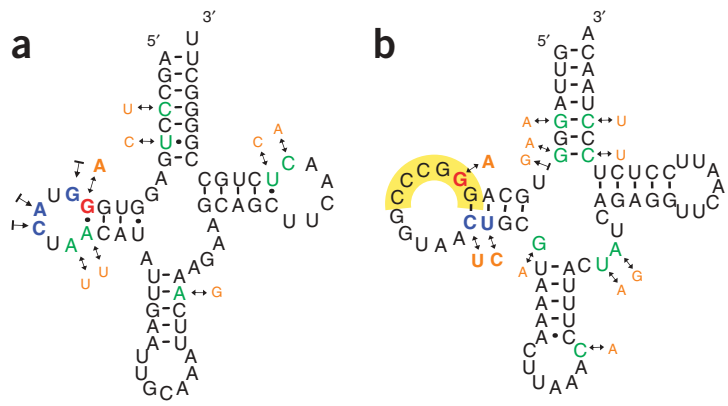

Figure 3 Secondary structures with interactions between loops and stems. (a) O. cuniculus (rabbit) tRNACys. (b) C. familiaris (dog) tRNA Leu (UUR). Known CPDs are shown in red; compensatory substitutions are shown in blue; and nucleotide states found in human are shown in orange. The pathogenic mutation in the tRNA ${ }^{\text {Cys }} \mathrm{D}$ stem is compensated in rabbit by the elimination of a hairpin loop found in humans. The human pathogenic mutation in tRNA Leu (UUR) completes a palindrome (yellow shading) that is prone to the formation of a dimer between two tRNALeu (UUR) molecules by partial unbinding of the $D$ stem ${ }^{15,16}$. The compensatory substitutions increase the free energy of the stem, preventing the formation of the dimer ${ }^{16}$. 

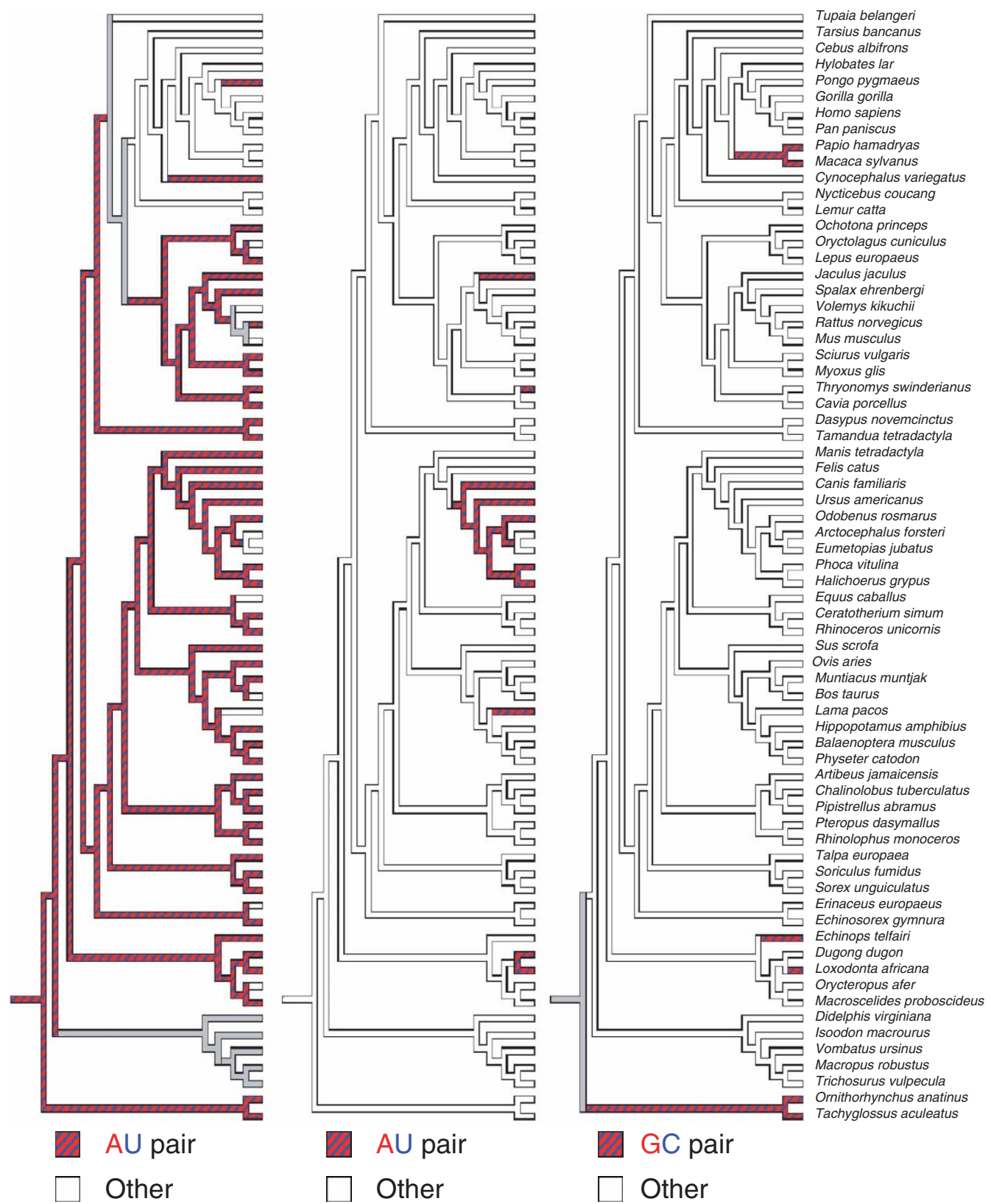

Figure 4 Convergent evolution of compensation of three pathogenic mutations, A26G in the anticodon stem of tRNA Asp (left), G46A in the TYC stem of tRNA ${ }^{\text {His }}$ (middle) and G67A in the acceptor stem of tRNA ${ }^{\text {Lys }}$ (right), in 69 mammalian species. CPDs are shown in red; compensatory substitutions are shown as blue lines; unresolved branches are shown in gray.

Note online). Thus, substitutions participating in compensatory interactions comprise $\sim 50 \%$ of all substitutions occurring in the evolution of mammalian tRNAs.

These findings show that the fitness surfaces along which mitochondrial tRNAs evolve consist of multiple highly epistatic ridges ${ }^{2,23}$. This implies that their fast rate of evolution is not an indication of mitochondrial mutational 'meltdown', or functional degradation, in an asexual genome ${ }^{24}$. The high frequency of convergent evolution and the observation that individual sites in tRNA genes often evolve nonindependently from each other suggest that RNA genes may be misleading when used for deep phylogenetic reconstruction ${ }^{22}$. Relating phenotypic, structural and evolutionary data provides invaluable information that can be used to understand the function and structural interactions of complex molecules. We suggest that similar approaches may facilitate the study of structural characteristics of tRNAs and provide further insight into the molecular basis of human disease.

\section{METHODS}

We obtained data on human disease-causing mutations from Mitomap and curated them manually to eliminate annotation errors in the database. We obtained 106 complete mitochondrial genomes from GenBank with the Entrez retrieval ${ }^{25}$ system using "mitochondrion AND complete AND genome AND mammal" as the search entry; only one genome for each species was used in 
our final data set, and the annotation for the genes was corrected when necessary using sequence similarity information with closely related species. We used a multiple alignment of 436 human complete mitochondria genomes to obtain polymorphism data in the human population. We constructed multiple alignments using CLUSTALW ${ }^{26}$ and manually corrected them using secondary structure information ${ }^{7}$. We used maximum parsimony to estimate the number of times CPDs and their predicted compensatory substitutions evolved on a mammalian phylogeny ${ }^{18}$. The use of different variants of the mammalian tree ${ }^{27}$ did not change our results. We used MacClade ${ }^{28}$ to draw the phylogenetic trees with CPDs and the RnaViz2 program ${ }^{29}$ to draw the tRNA secondary structures.

URLs. Mitomap is available at http://www.mitomap.org/.

Note: Supplementary information is available on the Nature Genetics website.

\section{ACKNOWLEDGMENTS}

We thank J. Gillespie, M. Hahn, L. Horth, A. Kondrashov, A. Kopp, S. Nuzhdin, M. Turelli and D. Weinreich for their contributions. The authors were supported by a grant from the US National Institutes of Health to S. Nuzhdin, and A.D.K. is a Howard Hughes Medical Institute predoctoral fellow.

\section{COMPETING INTERESTS STATEMENT}

The authors declare that they have no competing financial interests.

Received 14 June; accepted 20 September 2004

Published online at http://www.nature.com/naturegenetics/

1. Lodish, H. et al. in Molecular Cell Biology 265-266 (W.H. Freeman and Company, New York, 2000).

5 2. Kondrashov, A.S., Sunyaev, S. \& Kondrashov, F.A. Dobzhansky-Muller incompatibilities in protein evolution. Proc. Natl. Acad. Sci. USA. 99, 14878-14883 (2002).

3. Schon, E.A., Bonilla, E. \& DiMauro, S. Mitochondrial DNA mutations and pathogenesis. J. Bioenerg. Biomembr. 29, 131-149 (1997)

4. Andreu, A.L. \& DiMauro, S. Current classification of mitochondrial disorders. J. Neurol. 250, 1403-1406 (2003).

5. Gao, L.Z. \& Zhang, J.Z. Why are some human disease-associated mutations fixed in mice? Trends Genet. 19, 678-681 (2003).

6. Florentz, C. \& Sissler, M. Disease-related versus polymorphic mutations in human mitochondrial tRNAs. Where is the difference? EMBO Rep. 2, 481-486 (2001).

7. Helm, M. et al. Search for characteristic structural features of mammalian mitochondrial tRNAs. RNA 6, 1356-1279 (2000).

8. Zuker, M., Mathews, D.H. \& Turner, D.H. Algorithms and thermodynamics for RNA secondary structure prediction: a practical guide. in RNA Biochemistry and Biotechnology (eds. Barciszewski, J. \& Clark, B.F.C.) 1-23 (NATO ASI Series, Kluwer Academic, 1999).
9. Kimura, M. The role of compensatory neutral mutations in molecular evolution. J. Genet. 64, 7-19 (1985).

10. Kimura, M. Recent development of the neutral theory viewed from the Wrightian tradition of theoretical population genetics. Proc. Natl. Acad. Sci. USA. 88, 59695973 (1991).

11. Rousset, F., Pelandakis, M. \& Solignac, M. Evolution of compensatory substitutions through G.U intermediate state in Drosophila rRNA. Proc. Natl. Acad. Sci. USA 88 , 10032-10036 (1991).

12. Chen, Y. et al. RNA secondary structure and compensatory evolution. Genes Genet. Syst. 74, 271-286 (1999).

13. Parsch, J., Braverman, J.M. \& Stephan, W. Comparative sequence analysis and patterns of covariation in RNA secondary structures. Genetics 154, 909-921 (2000).

14. Kelley, S.O., Steinberg, S.V. \& Schimmel, P. Functional defects of pathogenic human mitochondrial tRNAs related to structural fragility. Nat. Struct. Biol. 7, 862-865 (2000).

15. Wittenhagen, L.M. \& Kelley, S.O. Impact of disease-related mitochondrial mutations on tRNA structure and function. Trends Biochem. Sci. 28, 605-611 (2003).

16. Wittenhagen, L.M. \& Kelley, S.O. Dimerization of a pathogenic human mitochondrial tRNA. Nat. Struct. Biol. 9, 586-590 (2002).

17. Higgs, P.G. Compensatory neutral mutations in the evolution of RNA. Genetica 102/103, 91-101 (1998).

18. Reyes, A. et al. Congruent mammalian trees from mitochondrial and nuclear genes using Bayesian methods. Mol. Biol. Evol. 21, 397-403 (2004).

19. Innan, H. \& Stephan, W. Selection intensity against deleterious mutations in RNA secondary structures and rate of compensatory nucleotide substitutions. Genetics 159, 389-399 (2001).

20. Gillespie, J.H. Variability of evolutionary rates of DNA. Genetics $113,1077-1091$ (1986).

21. Orr, H.A. \& Turelli, M. The evolution of postzygotic isolation: accumulating Dobzhansky-Muller incompatibilities. Evolution (Lawrence, Kans.) 55, 1085-1094 (2001).

22. Tillier, E.R. \& Collins, R.A. High apparent rate of simultaneous compensatory base-pair substitutions in ribosomal RNA. Genetics 148, 1993-2002 (1998).

23. Chao, L. \& Burch, C.L. Evolution by small steps and rugged landscapes in the RNA virus $\phi 6$. Genetics 151, 921-927 (1999).

24. Lynch, M. Mutation accumulation in nuclear, organelle, and prokaryotic transfer RNA genes. Mol. Biol. Evol. 14, 914-925 (1997).

25. Wheeler, D.L. et al. Database resources of the National Center for Biotechnology. Nucleic Acids Res. 31, 28-33 (2003).

26. Thompson, J.D., Higgins, D.G. \& Gibson, T.J. CLUSTAL W: improving the sensitivity of progressive multiple sequence alignment through sequence weighting, position-specific gap penalties and weight matrix choice. Nucleic Acids Res. 22, 4673-4680 (1994).

27. Murphy, W.J. et al. Molecular phylogenetics and the origins of placental mammals. Nature 409, 614-618 (2001).

28. Maddison, W.P. \& Maddison, D.R. MacClade: Analysis of Phylogeny and Character Evolution (Sinauer Associates, Sunderland, Massachusetts, 1992).

29. De Rijk, P., Wuyts, J. \& De Wachter, R. RnaViz2: an improved representation of RNA secondary structure. Bioinformatics 19, 299-300 (2003). 